\title{
Adubação do feijoeiro cultivado no norte de Minas Gerais com nitrogênio e fósforo ${ }^{1}$
}

\author{
Tarciana de Oliveira Viana², Neiva Maria Batista Vieira33, Guilherme Borém Lobato Moreira², Renata Oliveira \\ Batista $^{4}$, Saul Jorge Pinto de Carvalho ${ }^{3}$, Heverton Fernandes Ferreira Rodrigues ${ }^{4}$
}

\section{RESUMO}

Este trabalho foi realizado com o objetivo de avaliar a resposta da cultura do feijoeiro (Phaseolus vulgaris L), cv. Carioca Precoce, à adubação com nitrogênio $(\mathrm{N})$ e fósforo $(\mathrm{P})$, cultivado no norte de Minas Gerais. O experimento foi realizado na safra do inverno-primavera de 2008, com delineamento experimental de blocos casualizados e três repetições. Os tratamentos foram organizados segundo esquema fatorial 4x4, sendo quatro doses de N: 0, 70,140 e $210 \mathrm{~kg}$ ha${ }^{1}$ e quatro doses de P: 0, 100, 200 e $300 \mathrm{~kg} \mathrm{ha}^{-1} \mathrm{de}_{2} \mathrm{O}_{5}$. A ureia e o superfosfato simples foram utilizados como fontes de $\mathrm{N}$ e $\mathrm{P}$, respectivamente. $\mathrm{O}$ incremento das doses do fertilizante nitrogenado reduziu linearmente o estande inicial de plantas de feijão, sem influência das doses de fósforo. O número de vagens por planta foi o único componente de rendimento influenciado pela aplicação dos fertilizantes, unicamente para doses de $\mathrm{N}$, com número máximo na dose de $108 \mathrm{~kg} \mathrm{ha}^{-1}$ de $\mathrm{N}$. Houve resposta quadrática da produtividade de grãos às diferentes doses de $\mathrm{N}$ e $\mathrm{P}$, sendo a máxima produtividade $\left(1.528 \mathrm{~kg} \mathrm{ha}^{-1}\right)$ alcançada com $98 \mathrm{~kg} \mathrm{ha}^{-1}$ de $\mathrm{N}$ e $201 \mathrm{~kg} \mathrm{ha}^{-1}$ de $\mathrm{P}$, sem haver interação entre eles.

Palavras-chave: Phaseolus vulgaris L., feijão, nutrição, ureia, cultivo convencional.

\section{ABSTRACT}

\section{Nitrogen and phosphorous fertilization of common bean in Northern Minas Gerais State, Brazil}

This work was carried out to evaluate the common bean (Phaseolus vulgaris L.) cv. Carioca Precoce response to nitrogen $(\mathrm{N})$ and phosphorous $(\mathrm{P})$ fertilization, in the North Region of Minas Gerais. The trial was carried out in the winter-spring season, 2008, using a randomized block design with three replicates. Treatments were arranged in a $4 x 4$ factorial scheme, with four $\mathrm{N}$ rates: $0,70,140$ and $210 \mathrm{~kg} \mathrm{ha}^{-1}$; and four P rates : 0, 100, 200 and $300 \mathrm{~kg} \mathrm{ha}^{-1}$ of $\mathrm{P}_{2} \mathrm{O}_{5}$. Urea and simple superphosphate were used as $\mathrm{N}$ and $\mathrm{P}$ sources, respectively. Increasing $\mathrm{N}$ rates linearly reduced the initial stand of common bean, without influence of $\mathrm{P}$ rates. The number of pods per plant was the only yield component affected by fertilizer application, only for $\mathrm{N}$ rates, with the maximum number achieved at the rate of $108 \mathrm{~kg} \mathrm{ha}^{-1}$. The quadratic yield response was adjusted to the different rates of $\mathrm{N}$ and $\mathrm{P}$, with maximum yield $\left(1.528 \mathrm{~kg} \mathrm{ha}^{-1}\right)$ achieved with $98 \mathrm{~kg} \mathrm{ha}^{-1}$ of $\mathrm{N}$ and $201 \mathrm{~kg} \mathrm{ha}^{-1}$ of $\mathrm{P}$, without interaction between them.

Key words: Phaseolus vulgaris L., nutrition, urea, conventional crop.

\footnotetext{
Recebido para publicação em fevereiro de 2010 e aprovado em dezembro de 2010

'Monografia da $1^{\text {a }}$ autora, apresentada à UNIMONTES para obtenção do título de Engenheira-Agrônoma.

${ }^{2}$ Engenheira-Agrônoma, Depto. Ciências Agrárias, UNIMONTES, , Rua Reinaldo Viana, 2.630, Bico da Pedra, Janaúba-MG, 39.440-000. tarcianadeoliveira@yahoo.com.br; guiagronomia@hotmail.com

${ }^{3}$ Engenheira-Agrônoma, DS. Instituto Federal de Educação, Ciência e Tecnologia do Sul de Minas Gerais, CGPP, Caixa Postal 1.004, 37.750-971, Machado-MG sjpcarvalho@yahoo.com.br; neivavieira2003@yahoo.com.br*Autor correspondente

${ }^{4}$ Acadêmico em Agronomia. Depto. Ciências Agrárias, UNIMONTES, Rua Reinaldo Viana, 2.630, Bico da Pedra, Janaúba-MG, 39.440-000. renata_agro@yahoo.com.br; nandoheverton@hotmail.com
} 


\section{INTRODUÇÃO}

A literatura é relativamente rica em resultados sobre a aplicação de nitrogênio e fósforo no feijoeiro comum (Phaseolus vulgaris L.), permitindo concluir que esses são os nutrientes aos quais a cultura apresenta as maiores respostas. Entretanto, constata-se que, com algumas exceções (Rodrigues et al., 2002; Kikuti et al., 2005), a maioria desses trabalhos estudou separadamente os efeitos da adubação nitrogenada ou fosfatada, sem explorar satisfatoriamente a sua interação.

Em geral, as recomendações oficiais de adubação nitrogenada na cultura do feijoeiro para o Estado de Minas Gerais adotam doses entre 20 e $100 \mathrm{~kg} \mathrm{ha}^{-1}$ (Chagas et al., 1999), variando em razão da tecnologia empregada. Segundo Malavolta (1980), essa recomendação de adubação deve-se à baixa exigência inicial de nitrogênio $(\mathrm{N})$ pelas culturas anuais, ao efeito salino sobre a semente e à possibilidade de perdas por lixiviação.

Com relação aos componentes de produção do feijoeiro, de maneira geral, não são observados efeitos de doses de N, com exceção do número de vagens por planta, que possui incremento linear em razão da maior disponibilidade do nutriente (Soratto et al., 2004; Crusciol et al., 2007). Por outro lado, com frequência, doses crescentes de N têm provocado redução linear na população de plantas (Rodrigues et al., 2002; Kikuti et al., 2005). Sobre a produtividade, respostas do feijoeiro à adubação nitrogenada têm sido observadas em todo o Brasil, com intensidade e amplitude variáveis (Silveira \& Damasceno, 1993; Silva et al., 2000; Silveira et al., 2003; Soratto et al., 2004; Barbosa Filho et al., 2005; Meira et al., 2005; Crusciol et al., 2007).

O fósforo (P), por sua vez, é um elemento pouco móvel no solo e seu suprimento para as raízes é efetuado principalmente pelo processo de difusão em curta distância, o qual depende da umidade do solo e da superfície radicular (Gahoonia et al., 1994). O baixo teor de fósforo disponível no solo é a limitação nutricional mais generalizada na produção agrícola nos trópicos, sendo o nutriente que mais influencia na produtividade do feijoeiro na maioria das áreas brasileiras (Arf, 1994). No entanto, a eficiência da adubação fosfatada é baixa, visto que grande parte do $\mathrm{P}$ adicionado torna-se imóvel ou não disponível, em virtude de reações de adsorção em coloides minerais, precipitação ou conversão em formas orgânicas (Holford, 1997).

Poucos são os resultados encontrados para a resposta da população de plantas às doses de $\mathrm{P}$, já que os adubos que contêm este nutriente não possuem o poder salino verificado para os adubos que contêm nitrogênio. Com relação à produtividade, Silva et al. (2001), trabalhando em solo arenoso, obtiveram valores máximos com $104 \mathrm{~kg} \mathrm{ha}^{-1}$ de P. Em contrapartida, Silva \& Vahl
(2002) e Kikuti et al. (2005), em solos argilosos, obtiveram a máxima produtividade com doses bem mais altas, entre 225 e $251 \mathrm{~kg} \mathrm{ha}^{-1}$ de $\mathrm{P}$.

Trabalhando com os cultivares Carioca e Pérola submetidos a doses de $\mathrm{Ne}_{2} \mathrm{O}_{5}$, Rodrigues et al. (2002) encontraram resposta linear do feijoeiro ao $\mathrm{N}$, porém variável com a dose de fósforo. $\mathrm{O}$ efeito das doses de $\mathrm{P}_{2} \mathrm{O}_{5}$ foi significativo, linear e crescente, na presença e na ausência de N. Ainda, Kikuti et al. (2005) constataram máxima produtividade para 'BRS-MG Talismã' com doses percentuais maiores que as oficialmente recomendadas para Minas Gerais ( de 38 a 54\% para N e de 26 a $116 \%$ para $\mathrm{P})$.

De forma geral, muitas dúvidas ainda permanecem para a implantação do manejo racional da adubação do feijoeiro. Este trabalho foi realizado com o objetivo de avaliar a resposta da cultura do feijoeiro-comum à adubação nitrogenada e fosfatada, em plantio irrigado, na região norte de Minas Gerais.

\section{MATERIAL E MÉTODOS}

O experimento foi instalado na fazenda experimental da Universidade Estadual de Montes Claros, Campus Janaúba (4316'18'” W, 1549' 51" S e 540 m de altitude), localizada na região norte do Estado de Minas Gerais, na safra do inverno-primavera de 2008. O clima foi classificado como Aw, segundo classificação de Köpen, com precipitação média anual de $870 \mathrm{~mm}$. O solo da área foi classificado como Latossolo Vermelho eutrófico (Embrapa, 2006), de textura média. A análise química do solo está apresentada na Tabela 1, para amostras coletadas à profundidade de 0 a 0,20 m, antes da semeadura do feijão.

O cultivar de feijão utilizado foi o Carioca, de ciclo precoce, sendo bastante utilizado na região do Vale do Gorutuba, norte de Minas Gerais. O preparo do solo contou com uma aração e uma gradagem, em profundidade

Tabela 1. Análise química do solo da área experimental ( de 0 a $0,20 \mathrm{~m})$

\begin{tabular}{|c|c|}
\hline Características & Valores \\
\hline $\mathrm{pH}$ em $\mathrm{H}_{2} \mathrm{O}$ & 5,0 AcE \\
\hline $\mathrm{P}$ (Mehlich 1) $\mathrm{mg} \mathrm{dm}^{-3}$ & $19,4 \mathrm{~B}$ \\
\hline $\mathrm{K}$ (Mehlich 1) $\mathrm{mg} \mathrm{dm}^{-3}$ & $86,0 \mathrm{~B}$ \\
\hline $\mathrm{Ca} \quad \mathrm{cmolc} \mathrm{dm}^{-3}$ & $2,3 \mathrm{M}$ \\
\hline $\mathrm{Mg} \mathrm{cmolc} \mathrm{dm}^{-3}$ & $0,3 \mathrm{Ba}$ \\
\hline Al cmolc dm ${ }^{-3}$ & $0,0 \mathrm{MBa}$ \\
\hline Matéria Orgânica dag kg-1 & $1,1 \mathrm{Ba}$ \\
\hline $\mathrm{V}(\%)$ & $69,0 \mathrm{~B}$ \\
\hline Areia dag $\mathrm{kg}^{-1}$ & 48,0 \\
\hline Silte dag $\mathrm{kg}^{-1}$ & 23,0 \\
\hline Argila dag $\mathrm{kg}^{-1}$ & 29,0 \\
\hline
\end{tabular}

* Interpretação de acordo com Chagas et al. (1999). AcE = acidez elevada, $\mathrm{MBa}=$ muito baixo, $\mathrm{Ba}=$ baixo, $\mathrm{M}=$ médio, $\mathrm{B}=$ bom. 
média de $0,20 \mathrm{~m}$. A semeadura foi realizada manualmente, em 21/08/2008, adotando-se o espaçamento de 0,5 m entre linhas, densidade populacional de 240.000 plantas por hectare e profundidade de semeadura de 3,0 a $4,0 \mathrm{~cm}$.

Cada parcela foi constituída por quatro linhas de $5 \mathrm{~m}$ de comprimento, totalizando $10 \mathrm{~m}^{2}$ de área total e $5 \mathrm{~m}^{2} \mathrm{de}$ área útil. Todas as parcelas receberam idêntica adubação potássica (20 kg ha-1 de $\mathrm{K}_{2} \mathrm{O}$ ), determinada por meio do resultado de análise do solo (Tabela 1) e da sua interpretação segundo Chagas et al. (1999). A correção do solo não foi necessária, visto que a ocorrência de saturação de bases da ordem de $69 \%$ é considerada boa para a cultura do feijoeiro.

O delineamento experimental utilizado foi o de blocos casualizados, com três repetições. Os tratamentos foram organizados segundo esquema fatorial $4 \times 4$, com quatro doses de nitrogênio: $0,70,140$ e $210 \mathrm{~kg} \mathrm{ha}^{-1}$ e quatro doses de fósforo: $0,100,200$ e $300 \mathrm{~kg} \mathrm{ha}^{-1} \mathrm{de}_{2} \mathrm{O}_{5}$. A ureia e o superfosfato simples foram utilizados como fonte de nitrogênio e de fósforo, respectivamente. $\mathrm{O}$ nitrogênio foi parcelado em duas aplicações, sendo $50 \%$ na semeadura e $50 \%$ em cobertura no estádio $\mathrm{V}_{3}-\mathrm{V}_{4}(\mathrm{Gepts} \&$ Fernández, 1982), aos 25 dias após emergência (DAE). Os demais tratos culturais foram aqueles normalmente dispensados à cultura na região. $\mathrm{O}$ experimento foi irrigado por aspersão convencional.

Aos 30 DAE, foi avaliada a germinação das plantas, mediante contagem do estande inicial; para tanto, todas as plantas das duas linhas centrais das parcelas foram contabilizadas. Por ocasião da colheita (estádio $\mathrm{R}_{9}$ ), após ciclo de 79 dias, avaliaram-se: produtividade de grãos a $13 \%$ de umidade e os componentes do rendimento (número de vagens por planta, número de grãos por vagem e massa de 100 grãos). A produtividade de grãos foi avaliada mediante colheita de todas as plantas pertencentes às duas linhas centrais de cada parcela, sendo dez plantas selecionadas, aleatoriamente, para avaliação das demais características.

Os dados foram submetidos à aplicação do teste $\mathrm{F}$ na análise da variância, com significância de 5\%. Quando significativos, os efeitos quantitativos foram analisados por meio de regressões polinomiais. A produtividade de plantas $\left(\mathrm{kg} \mathrm{ha}^{-1}\right)$ foi transformada por $\sqrt{x+0,5}$. As máximas produtividades de grãos e os componentes de rendimento foram estimados por meio da primeira derivada do modelo quadrático. As análises estatísticas foram realizadas com auxílio do programa Sisvar ${ }^{\circledR}$ (Ferreira, 2000).

\section{RESULTADOS E DISCUSSÃO}

Verificou-se efeito significativo das doses de nitrogênio sobre as variáveis estande inicial, número de vagens por planta e produtividade de grãos. As diferentes doses de fósforo utilizadas influenciaram apenas a produtividade. Não houve interação das doses de N e P para quaisquer das variáveis avaliadas.

$\mathrm{O}$ aumento das doses de $\mathrm{N}$ diminuiu linearmente o estande inicial do feijoeiro (Figura 1), com redução da ordem de 280 plantas ha ${ }^{-1}$ para cada quilo de nitrogênio aplicado por hectare, em população de 240 mil plantas ha${ }^{1}$. Assim, torna-se evidente o efeito negativo do aumento da dose de $\mathrm{N}$ em semeadura sobre o estande inicial, que é consequência do efeito salino do fertilizante sobre a germinação das sementes (Rodrigues et al., 2002; Kikuti et al., 2005). Esse argumento é altamente relevante para o presente trabalho, em que a dose máxima de nitrogênio utilizada foi de $210 \mathrm{~kg} \mathrm{ha}^{-1}$, com aplicação de $105 \mathrm{~kg} \mathrm{ha}^{-1}$ na semeadura.

Pode ser observado, também, que apesar de se tratar de experimento desenvolvido sob irrigação, essa não foi suficiente para amenizar o efeito salino do fertilizante na germinação das sementes, evidenciado pelaa redução populacional. Entretanto, as populações observadas, mesmo nas doses mais altas de nitrogênio, mantiveramse dentro das recomendações usuais (Del Peloso, 1996). Todavia, cuidados devem ser tomados na relação entre estande inicial e produtividade, com necessidade de verificar se o menor número de plantas por hectare tem reflexo em menor produtividade.

Observou-se ajuste quadrático do número de vagens por planta aos níveis de fertilização com N (Figura 2). O maior número de vagens por planta foi verificado na dose de $108 \mathrm{~kg} \mathrm{ha}^{-1}$ de $\mathrm{N}$, alcançando a produtividade de 14 vagens por planta. Esse resultado foi diferente daqueles encontrados por Soratto et al. (2004) e Crusciol et al. (2007), cuja resposta foi linear positiva (120 e $210 \mathrm{~kg} \mathrm{~N} \mathrm{ha}^{-1}$ ) e Meira et al. (2005), que não observaram efeito sobre esta variável, em cultivo sob plantio convencional. Por outro lado, Piaskowski et al. (2001) encontraram resultados superiores aos deste trabalho para dose de $80 \mathrm{~kg} \mathrm{ha}^{-1}$ de $\mathrm{N}$, em plantio direto. Supostamente, essas diferenças podem

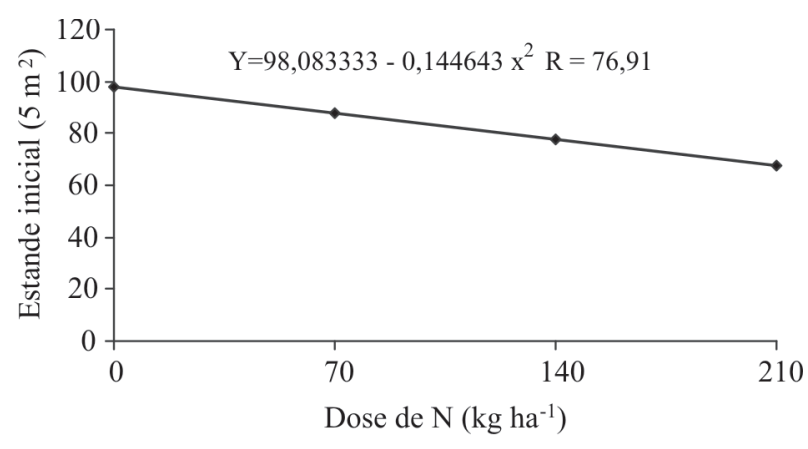

Figura 1. Estande inicial do feijoeiro cv. Carioca Precoce em função das diferentes doses de nitrogênio, na safra invernoprimavera.

Rev. Ceres, Viçosa, v. 58, n.1, p. 115-120, jan/fev, 2011 
ser creditadas às condições edafoclimáticas diferenciadas em que os experimentos foram desenvolvidos.

Portes (1996) constatou que plantas de feijão bem nutridas produzem mais flores e, consequentemente, mais vagens por planta. Entretanto, altas doses de N, assim como a variável estande inicial, resultaram em efeito negativo, provavelmente, pelo mesmo motivo já discutido anteriormente.

O número de vagens por planta é o componente primário que mais se correlaciona com a produtividade de grãos (Silva et al., 2007; Souza et al., 2008) e é bastante influenciado pelo ambiente (Arf et al., 1996; Thomas, 2000). As doses de $\mathrm{P}$ aplicadas ao solo não influenciaram o estande inicial e o número de vagens por planta (Tabela 3). Esses dados corroboram aqueles encontrados por Rodrigues et al. (2002) e Kikuti et al. (2005). Entretanto, esses autores encontraram efeito significativo da interação $\mathrm{NxP}$, fato não observado neste trabalho. O número de grãos por vagem e massa de 100 grãos não foram influenciados pelas doses de $\mathrm{N}$ e $\mathrm{P}$ (Tabelas 2 e 3); resultados semelhantes foram encontrados por Soratto et al. (2004), Arf et al. (2004) e Meira et al. (2005), estudando doses de $\mathrm{N}$ na cultura do feijoeiro.

Os resultados de produtividade foram bastante superiores à média nacional $\left(893 \mathrm{~kg} \mathrm{ha}^{-1}\right)$ e semelhantes à

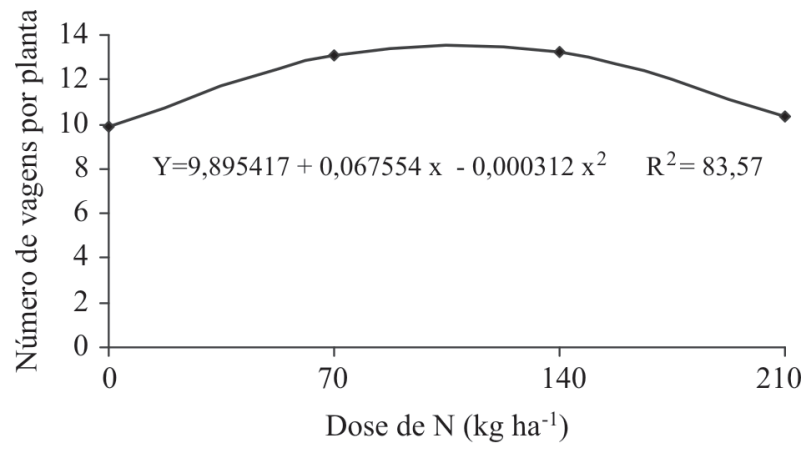

Figura 2. Número de vagens por planta de feijoeiro cv. Carioca Precoce em função das diferentes doses de nitrogênio, safra inverno-primavera.

Tabela 2. Médias do número de grãos por vagens $(\mathrm{G} / \mathrm{V})$ e massa de 100 grãos (M 100) de plantas de feijoeiro (cv. Carioca Precoce) em função das diferentes doses de nitrogênio, safra de invernoprimavera

\begin{tabular}{lcc}
\hline Dose de $\mathbf{N}\left(\mathbf{k g ~ h a}^{-1}\right)$ & G/V & M100(g) \\
\hline 0 & 5,2 & 18,8 \\
70 & 4,1 & 19,8 \\
140 & 4,1 & 20,2 \\
210 & 3,8 & 19,8 \\
$\mathrm{~F}_{\text {(nitrogênio) }}$ & $1,84^{\mathrm{ns}}$ & $0,81^{\mathrm{ns}}$ \\
$\mathrm{F}_{\text {(interaç̃o) }}$ & $0,49^{\mathrm{ns}}$ & $1,13^{\mathrm{ns}}$ \\
\hline
\end{tabular}

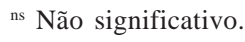

Tabela 3. Médias do estande inicial (EI), número de vagens por planta (V/P), número de grãos por vagens $(\mathrm{G} / \mathrm{V})$ e massa de 100 grãos (M 100) de plantas de feijoeiro cv. Carioca Precoce em função das diferentes doses de fósforo, safra de inverno-primavera

\begin{tabular}{lcccc}
\hline Dose de $\mathrm{P}\left(\mathrm{kg} \mathrm{ha}^{-1}\right)$ & EI & V/P & G/V & M 100(g) \\
\hline 0 & 67 & 10,2 & 4,3 & 19,0 \\
100 & 83 & 12,2 & 4,1 & 20,9 \\
200 & 98 & 14,2 & 4,8 & 19,2 \\
300 & 83 & 10,0 & 3,9 & 19,5 \\
$\mathrm{~F}_{\text {(f́śforo) }}$ & $2,17^{\mathrm{ns}}$ & $2,53^{\mathrm{ns}}$ & $0,72^{\mathrm{ns}}$ & $3,03^{\mathrm{ns}}$ \\
$\mathrm{F}_{\text {(interaçăo) }}$ & $0,69^{\mathrm{ns}}$ & $1,12^{\mathrm{ns}}$ & $0,49^{\mathrm{ns}}$ & $1,13^{\mathrm{ns}}$ \\
\hline
\end{tabular}

ns Não significativo.

média mineira (1.453 $\left.\mathrm{kg} \mathrm{ha}^{-1}\right)$, tanto para os efeitos isolados das doses de $\mathrm{N}$, quanto para doses de $\mathrm{P}$ (CONAB, 2009) (Figuras 3 e 4). A maior produtividade de grãos (1.528 $\mathrm{kg} \mathrm{ha}^{-1}$ ) foi alcançada com a dose de $98 \mathrm{~kg} \mathrm{ha}^{-1}$ de N (Figura 3). Esse resultado é superior aos observados por Carvalho et al. (1992), Silveira \& Damasceno (1993) e Silva et al. (2000), em plantio convencional e Silveira et al. (2003), em plantio direto.

Por outro lado, Soratto et al. (2004) e Barbosa Filho et al. (2005) encontraram doses correspondentes à produtividade máxima de grãos muito maiores que as detectadas

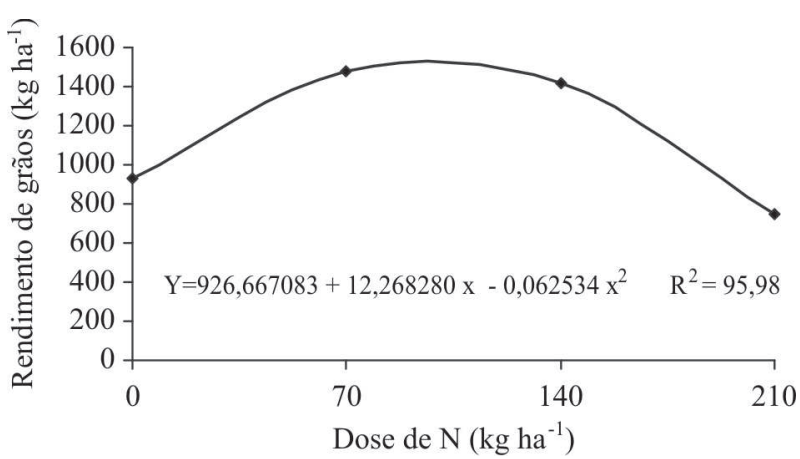

Figura 3. Produtividade $\left(\mathrm{kg} \mathrm{ha}^{-1}\right)$ de planta de feijoeiro cv. Carioca Precoce em função das diferentes doses de nitrogênio, safra inverno-primavera.

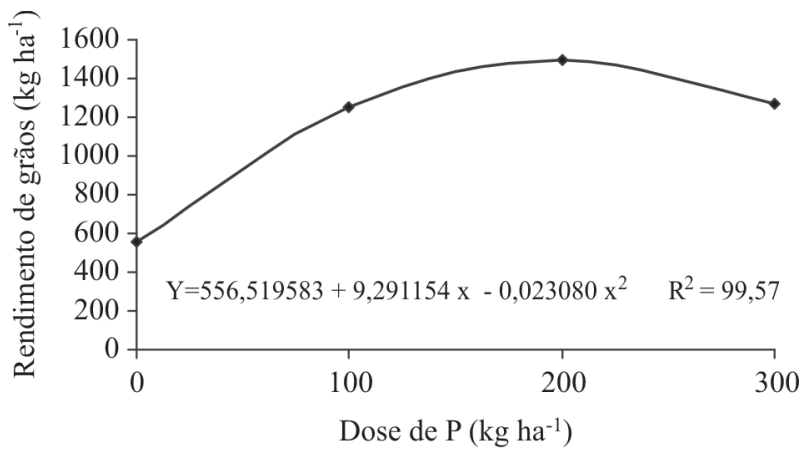

Figura 4. Produtividade $\left(\mathrm{kg} \mathrm{ha}^{-1}\right)$ de plantas de feijoeiro cv. Carioca Precoce em função das diferentes doses de nitrogênio, safra inverno-primavera.

Rev. Ceres, Viçosa, v. 58, n.1, p. 115-120, jan/fev, 2011 
neste trabalho, com produtividades também bastante superiores. Ainda, Soratto et al. (2001) e Crusciol et al. (2007) encontraram produtividades máximas com doses bastante superiores às encontradas no presente trabalho, em plantio direto.

A produtividade de grãos em função de doses de P está apresentada na Figura 4, com efeito significativo em modelo quadrático. A máxima produtividade $\left(1.492 \mathrm{~kg} \mathrm{ha}^{-1}\right)$ ocorreu na dose de $201 \mathrm{~kg} \mathrm{ha}^{-1}$ de $\mathrm{P}_{2} \mathrm{O}_{5}$, bem superior àquelas normalmente recomendadas para a cultura no Estado (Chagas et al., 1999). Esse resultado é bastante semelhante aos encontrados por Silva \& Vahl (2002) e Kikuti et al. (2005), entretanto, superiores aos encontrados por Fageria \& Santos (1998) e Silva et al. (2001), em solo arenoso. Essa divergência de resultados deve-se, principalmente, à disponibilidade de $\mathrm{P}\left(19,4 \mathrm{mg} \mathrm{dm}^{-3}\right.$, considerado bom) $\mathrm{e}$ presença de água no solo, além da textura. Segundo Miranda et al. (2002), o feijoeiro responde significativamente à adubação fosfatada no sulco de plantio, dependendo da disponibilidade inicial de P. De maneira geral, solos arenosos e com maior teor de $\mathrm{P}$ tendem a alcançar máximas produtividades com doses menores de $\mathrm{P}$, provavelmente em decorrência da menor fixação deste elemento (Fageria \& Santos, 1998; Silva et al., 2001).

A maior necessidade de $\mathrm{P}$ encontrada neste trabalho pode ser explicada pela baixa disponibilidade de água (safra inverno-primavera), apesar da presença de irrigação, que favorece a disponibilidade de $\mathrm{P}$ às plantas (Miranda et al., 2000). Mais uma vez, as condições edafoclimáticas podem ser as responsáveis por essa diferença, além da ausência de correção do solo.

\section{CONCLUSÕES}

$\mathrm{O}$ incremento das doses do fertilizante nitrogenado reduziu o estande inicial de plantas de feijão.

O número de vagens por planta foi afetado pelas diferentes doses de nitrogênio, com ponto máximo na dose de $108 \mathrm{~kg} \mathrm{ha}^{-1}$ de $\mathrm{N}$.

A máxima produtividade do feijoeiro foi alcançada com aplicação de $98 \mathrm{~kg} \mathrm{ha}^{-1}$ de $\mathrm{N}$ e de $201 \mathrm{~kg} \mathrm{ha}^{-1}$ de P.

Não se verificou interação entre N e P.

\section{REFERÊNCIAS}

Arf O (1994) Importância da adubação na qualidade do feijão e caupi. In: Sá ME \& Buzetti S. Importância da adubação na qualidade dos produtos agrícolas. São Paulo. p.233-248.

Arf O, Sá ME, Okita CS, Tiba MA, Guerreiro Neto G \& Ogassawara O (1996) Efeito de diferentes espaçamentos e densidades de semeadura sobre o desenvolvimento do feijoeiro (Phaseolus vulgaris L.). Pesquisa Agropecuária Brasileira, 31(9):629-634.

Arf O, Rodrigues RAF, Sá ME, Buzetti S \& Nascimento V (2004) Manejo do solo, água e nitrogênio no cultivo de feijão. Pesquisa Agropecuária Brasileira, 39(2):131-138.
Barbosa Filho MP, Fageria N \& Silva OF (2005) Fontes, doses e parcelamento da adubação nitrogenada em cobertura para feijoeiro comum irrigado. Ciência e Agrotecnologia, 29(1):69-76.

Carvalho AM, Silva AM, Costa EF \& Couto L (1992) Influência da fertirrigação no rendimento de grãos e componentes de produção do feijoeiro comum (Phaseolus vulgaris L.) cv. Carioca. Ciência e Prática, 16:503-511.

Chagas JM, Braga JM, Vieira C, Salgado LT, Junqueira Neto A, Araújo GAA, Andrade MJB, Lana RMQ \& Ribeiro AC (1999) Feijão. In: Ribeiro AC, Guimarães PTG, Alvarez VH (Eds.) Recomendações para o uso de corretivos e fertilizantes em Minas Gerais - 5 Aproximação. p. 306-307.

CONAB - Companhia Nacional de Abastecimento (2009) Levantamento de grãos 2009/2010. Disponível em: <http:// www.conab.gov.br/conabweb/download/safra/ 4graos_07.01.10.pdf>. Acessado em: 20 de dezembro de 2010.

Crusciol CAC, Soratto RP, Silva LM, Lemos LB (2007) Fontes e doses de nitrogênio para o feijoeiro em sucessão a gramíneas no sistema plantio direto. Revista Brasileira de Ciência do Solo, 31(6): 1545-1552.

Del Peloso MJ (1996) Cultivo irrigado em terras altas. In: Araújo RS, Rava CA, Stone LF \& Zimmermann MJ (Eds.). Cultura do feijoeiro comum no Brasil. Potafós. p. 517-584.

Empresa Brasileira de Pesquisa Agropecuária. Centro Nacional de Pesquisa de Solos. Sistema brasileiro de classificação de solos. 2.ed. (2006) Rio de Janeiro. 306p.

Fageria, NK \& Santos, AB (1998) Adubação fosfatada para o feijoeiro em solo de várzea. Revista Brasileira de Engenharia Agrícola e Ambiental, 2(2):124-127.

Ferreira D F (2000) Análises estatísticas por meio do Sisvar para Windows versão 4.0. p. 255-258. In: Reunião Anual da Região Brasileira da Sociedade Internacional de Biometria. Anais, São Carlos UFSCAR, 45.

Gahoonia TS, Raza S \& Nielsen NE (1994) Phosphorus de Pletion in the Rizosphere as Influenced by Soil Moisture. Plant And Soil, 159(2): 213-218.

Geptz P \& Fernandéz F (1982) Etapas de desarrollo de la planta de frijol comum (Phaseolus vulgaris L.). Cali, Colombia, CIAT. 10p. (mimeografado).

Holford ICR (1997) Soil Phosphorus: its measurement, and its uptake by plants. Journal of Soil Research, 35:227-239.

Kikuti H, Andrade MJB, Carvalho, JG \& Morais, AR (2005) Nitrogênio e fósforo em feijão (Phaseolus vulgaris L.) variedade cultivada BRS MG Talismã. Acta Scientiarum Agronomy, 27(3):415-422.

Malavolta E (1980) Elementos de Nutrição Mineral de Plantas. Piracicaba, Agronômica Ceres, 251p.

Meira FA, Sá ME, Buzetti S \& Arf O (2005) Doses e épocas de aplicação de nitrogênio no feijoeiro irrigado cultivado em plantio direto. Pesquisa Agropecuária Brasileira, 40(4):383-388.

Miranda LN, Azevedo JA, Miranda JCC \& Gomes AC (2000) Produtividade do feijoeiro em resposta à adubação fosfatada e regimes de irrigação em solo de cerrado. Pesquisa Agropecuária Brasileira, 35(4):703-710.

Miranda LN, Azevedo JA, Miranda JCC \& Gomes AC (2002) Calibração de métodos de análise de fósforo e resposta do feijão ao fósforo no sulco. Pesquisa Agropecuária Brasileira, 37(11):1621-1627.

Piaskowski SR, Júnior PR, Daros E \& Koehler HS (2001) Adubação nitrogenada em cobertura para o feijoeiro em plantio direto na palha. Scientia Agraria, 2(1-2):67-72. 
Portes TA (1996) Ecofisiologia. In: Araújo RS, Rava CA, Stone LF \& Zimmermann MJ (Eds.). Cultura do feijoeiro comum no Brasil. Piracicaba, Potafós. p. 101-137.

Rodrigues JRM, Andrade MJB, Carvalho, JG, Morais AR \& Rezende PM (2002) População de plantas e rendimento de grãos do feijoeiro em função de doses de nitrogênio e fósforo. Ciência e Agrotecnologia, 26(6):1218-1227.

Silva AO, Lima EA \& Menezes HEA (2007) Rendimento de grãos de feijão (Phaseolus vulgaris L.), cultivado em diferentes densidades de plantio. Revista das Faculdades Integradas de Bebedouro, 3:1-5.

Silva RJS \& Vahl LC (2002) Resposta do feijoeiro à adubação fosfatada num Neosolo Litolítico distrófico da região sul do Rio Grande do Sul. Revista Brasileira de Agrociência, 8(2): 129-132.

Silva TRB, Soratto RP, Chidi SN, Arf O, Sá ME \& Buzetti S (2000). Doses e aplicação de nitrogênio em cobertura na cultura do feijoeiro de inverno. Cultura Agronômica, 9:1-17.

Silva E B, Resende JCF \& Cintra WR (2001) Resposta do feijoeiro a doses de fósforo em solo arenoso. Ciência Rural, 31(6):973-977.
Silveira PM \& Damasceno MA (1993) Doses e parcelamento de K e $\mathrm{N}$ na cultura do feijoeiro irrigado. Pesquisa Agropecuária Brasileira, 28(11):1269-1276.

Silveira PM, Braz AJBP \& Didonet AD (2003) Uso de clorofilômetro como indicador da necessidade de adubação nitrogenada em cobertura no feijoeiro. Pesquisa Agropecuária Brasileira, 38(9):1083-1087.

Soratto RP, Silva TRB, Arf O \& Carvalho MAC (2001) Níveis e épocas de aplicação de nitrogênio em cobertura no feijoeiro irrigado em plantio direto. Cultura Agronômica, 10:89-99.

Soratto RP, Carvalho MAC \& Arf O (2004) Teor de clorofila e produtividade do feijoeiro em razão da adubação nitrogenada. Pesquisa Agropecuária Brasileira, 39(9):895-901.

Souza AB, Andrade MJB, Vieira NMB \& Albuquerque A (2008) Densidades de semeadura e níveis de NPK e calagem na produção do feijoeiro sob plantio convencional. Pesquisa Agropecuária Tropical, 38(2):39-43.

Thomas LF (2000) População de plantas para feijoeiro-comum (Phaseolus vulgaris L.) na safrinha em Santa Maria-RS. Dissertação de Mestrado, Universidade Federal de Santa Maria, Santa Maria. 129 p. 